\title{
Dunkle Gewalten.
}

\author{
Opifdip Didflungen
}

Dort

\section{Germann Rings.}

\section{Stuttgart.}

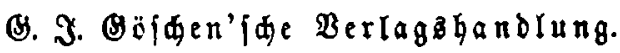

1872. 
Drud bon 9 . (9. Teubner in Leipjig. 